\title{
Mitotic arrest deficiency 2 induces carcinogenesis in mucinous ovarian tumors
}

\author{
YUSUKE NAKANO, TOSHIYUKI SUMI, MASANARI MORISHITA, TAKESHI FUKUDA, HIROYUKI NOBEYAMA, \\ HIROYUKI YOSHIDA, YOSHINARI MATSUMOTO, TOMOYO YASUI and OSAMU ISHIKO
}

Department of Obstetrics and Gynecology, Osaka City University Graduate School of Medicine, Asahimachi, Abeno-ku, Osaka 545-8585, Japan

Received July 20, 2011; Accepted October 18, 2011

DOI: $10.3892 / \mathrm{ol} .2011 .483$

\begin{abstract}
Mitotic arrest deficiency 2 (MAD2) is a key component of the mitotic spindle checkpoint pathway. A compromised mitotic spindle checkpoint results in an abnormal number of chromosomes. This is referred to as chromosomal instability, and has been reported in most types of human cancer. The aim of this study was to examine the expression of MAD2 in mucinous ovarian tumors exhibiting varying degrees of malignancy. We reviewed 128 cases of mucinous ovarian tumors initially treated at Osaka City University Medical School Hospital, Japan. Tumor samples were obtained following surgery. The cases were divided into three groups: benign (group B; $\mathrm{n}=30$ ), borderline malignant (group BM; $\mathrm{n}=55$ ) and malignant (group $\mathrm{M} ; \mathrm{n}=43$ ). MAD2 expression was examined in paraffin-embedded sections using the avidin-biotin peroxidase complex method. Results showed MAD2 expression to be significantly greater in group $\mathrm{M}$ compared to groups $\mathrm{B}$ and $\mathrm{BM}(\mathrm{P}<0.05)$. In addition, there was a moderate correlation between MAD2 expression and the degree of malignancy $(r=0.51, P<0.05)$. However, when the samples in group $M$ were classified according to a low or high expression of MAD2, no difference was observed in terms of overall survival. These findings suggest that the overexpression of MAD2 may be correlated to carcinogenesis in mucinous ovarian tumors.
\end{abstract}

\section{Introduction}

Mitotic arrest deficiency 2 (MAD2) was the first mammalian gene of the mitotic spindle checkpoint pathway to be characterized (1). MAD2 localizes at kinetochores following chromosome condensation and prior to anaphase (2), and plays a significant role in the transition from metaphase to anaphase by inhibiting the anaphase promoting complex/cyclosome

Correspondence to: Dr Toshiyuki Sumi, Department of Obstetrics and Gynecology, Osaka City University Graduate School of Medicine, 1-4-3 Asahimachi, Abeno-ku, Osaka 545-8585, Japan E-mail: sumi-toshi@med.osaka-cu.ac.jp

Key words: mitotic arrest deficiency 2, chromosomal instability, mucinous ovarian tumor, carcinogenesis
(APC/C). This process ensures that all the chromosomes are correctly aligned at the metaphase plate prior to daughter cell segregation $(3,4)$. Therefore, MAD2 is a key component of the mitotic spindle checkpoint pathway, which plays a crucial role in preventing loss or gain of chromosomes within cells (5). A compromised mitotic spindle checkpoint results in an abnormal number of chromosomes, known as chromosomal instability (CIN) (6). CIN, characterized by an alteration in chromosome number and commonly detected as aneuploidy $(7,8)$, has been reported in most types of human cancer. Although the underlying molecular mechanisms have yet to be clarified, it is notable that the overexpression of MAD2 in transgenic mice results in CIN, and initiates carcinogenesis in a wide variety of tumors (9). Another study showed that MAD2 is critical for the malignant transformation of epithelial cells, promoting aneuploidy and predisposing human prostate epithelial cells to carcinogen-induced malignant transformation (10). This finding suggests that a compromised mitotic spindle checkpoint, through the overexpression of MAD2, may be a significant step in the malignant progression of human cancer. Overexpression of MAD2 is observed in a variety of cancer (11-21). Certain reports suggested a correlation between the overexpression of MAD2 and a variety of clinicopathological characteristics, such as histological grade (differentiation), metastasis and prognosis (14-20). Almost all of these studies have concluded that the overexpression of MAD2 is a risk factor for poor prognosis. Therefore, the overexpression of MAD2 induces not only carcinogenesis, but also cancer progression.

Ovarian epithelial carcinoma is classified into four morphologically distinct categories: serous, mucinous, endometrioid and clear cell. Each category may be histopathologically sub-classified into benign, malignant, and borderline or low malignant potential (LMP). Mucinous tumors of the ovary account for $12-15 \%$ of all ovarian neoplasms, the majority being benign (approximately 75\%). Borderline and adenocarcinoma account for 10 and $15 \%$ of cases, respectively (22). Almost all mucinous borderline tumors are classified as stage I based on the International Federation of Gynecology and Obstetrics (FIGO) criteria and have an excellent prognosis following surgical treatment. The corrected actuarial survival rates are $98 \%$ at 5 years and $96 \%$ at 10 years $(23,24)$. Late-stage mucinous ovarian carcinomas are extremely 
rare and have a poor prognosis since they respond poorly to first-line platinum-based chemotherapy compared with other epithelial ovarian tumors (25). Mucinous ovarian tumors have unique clinicopathological characteristics associated with the development and progression of mucinous ovarian carcinoma. A number of studies have suggested that, unlike other histologic subtypes such as serous tumors, a proportion of mucinous ovarian carcinomas progress from a benign cyst to a borderline tumor prior to developing into a carcinoma. The coexistence of cells within mucinous ovarian tumors with varying degrees of malignancy increases the possibility of disease progression $(26,27)$. Previous findings have shown that a k-ras mutation may be an early event in mucinous ovarian tumorigenesis as the same k-ras mutations were observed in benign and borderline regions (27). Results of another study have indicated that benign tumors co-expressed a subset of genes that were differentially regulated in borderline tumors and carcinomas (28).

In this study, we examined the expression of MAD2 in mucinous ovarian tumors with varying degrees of malignancy.

\section{Materials and methods}

Patients and samples. We reviewed 128 cases of mucinous ovarian tumor initially treated at Osaka City University Medical School Hospital, Japan, between 1999 and 2010. Tumor samples were obtained following primary surgery. The cases were divided into three groups: benign (group B; $\mathrm{n}=30$ ), borderline malignant (group BM; $\mathrm{n}=55$ ) and malignant (group $\mathrm{M} ; \mathrm{n}=43$ ). Based on the FIGO criteria, the cases in group BM were classified as stage I $(n=52)$, stage II $(n=1)$, stage III $(n=2)$ and stage IV $(n=0)$, and the cases in group M as stage I $(n=25)$, stage II $(n=8)$, stage III $(n=8)$ and stage IV $(n=2)$ (Table I). Written informed consent was obtained from all patients prior to immunohistochemical examination, and this study was approved by the Ethics Committee of Osaka City University (IRB no. 2180).

Immunohistochemical analysis. Expression of MAD2 was investigated in paraffin-embedded sections using a MAD2 antibody and the avidin-biotin peroxidase complex method. Paraffin sections $(4-\mu \mathrm{m})$ were de-paraffinized and immersed in $3 \%$ hydrogen peroxidase in methanol to block endogenous peroxidase activity. An antigen retrieval procedure was then performed by immersing the slides in $10 \mathrm{mM}$ citrate buffer (pH 6.0) and heating the sections in an autoclave at $110^{\circ} \mathrm{C}$ for $20 \mathrm{~min}$. The sections were then washed in phosphate-buffered saline (PBS). The protocol for the Dako LSAB 2 peroxidase kit (Dako, Kyoto, Japan) was followed.

The sections were incubated with the primary antibodies for $2 \mathrm{~h}$ at room temperature. The primary antibody used for this study was monoclonal rabbit anti-human MAD2 (1:200; ProteinTech Group, Chicago, USA). Sections were rinsed with PBS for $15 \mathrm{~min}$ and incubated for $10 \mathrm{~min}$ with the secondary antibody (biotinylated goat anti-mouse and rabbit immunoglobulin G; Dako). The sections were incubated with the streptavidin-peroxidase complex and 3,3'-diaminobenzidine was used as the chromogen. The sections were then counterstained with Mayer's hematoxylin. The specificity of the immunohistochemical reactions was checked by omitting
Table I. Patient characteristics.

\begin{tabular}{lccc}
\hline & Group B & Group BM & Group M \\
\hline No. of cases & 30 & 55 & 43 \\
Age & & & \\
Mean & 52.5 & 47.3 & 53.4 \\
Range & $17-70$ & $13-88$ & $23-78$ \\
FIGO stage & & & \\
I & & 52 & 25 \\
& & $(94.5 \%)$ & $(58.1 \%)$ \\
II & 1 & 8 \\
& & $(1.8 \%)$ & $(18.6 \%)$ \\
III & 2 & 8 \\
& & $(3.8 \%)$ & $(18.6 \%)$ \\
IV & 0 & 2 \\
& & $(0 \%)$ & $(4.7 \%)$ \\
\hline
\end{tabular}

FIGO, International Federation of Gynecology and Obstetrics.

the primary antibody. Quantitative analysis of MAD2 expression was based on the scoring method of Sinicrope et al (29). The mean percentage of positive tumor cells was determined in five separate areas (magnification, x400) and assigned to one of the following categories: $0,<5 \% ; 1,5-25 \% ; 2,25-50 \%$; $3,50-75 \%$; or $4,>75 \%$. The intensity of immunostaining was scored as: $1+$, weak; $2+$, moderate; or $3+$, intense. For each specimen, the percentage of positive tumor cells was multiplied by the staining intensity to produce a weighted score.

Statistical analysis. The Kaplan-Meier and log-rank tests were used for the prognostic analyses. StatView 5.0 (Abacus Concepts, Berkley, CA, USA) was used for data analysis. Statistical significance was set at $<0.05$. Continuous variables were expressed as the mean \pm standard deviation (SD) or the mean \pm standard error (SE) as shown in the figures. Weighted scores were compared using the Mann-Whitney U test and Spearman's rank correlation coefficient.

\section{Results}

Patient characteristics. We reviewed 128 cases of mucinous ovarian tumors. Of the 128 cases, 30 cases were benign, 43 cases borderline malignant and 55 cases were malignant. The mean age group B was 52.5 years (range 17-70), that of group BM was 47.3 years (range 17-88), and that of group $M$ was 53.4 years (range 23-78). No significant difference was observed among the three groups (Table I). There were significantly more advanced cases in group $M$ than in group BM $(\mathrm{P}<0.05)$.

MAD2 expression. MAD2 was expressed in the nuclei of the tumor cells (Fig. 1). The mean weighted score of group B was 3.2, that of group BM was 4.3, and that of group $M$ was 7.6 (Table II). MAD2 expression was significantly greater in group $\mathrm{M}$ than in groups $\mathrm{B}$ and $\mathrm{BM}(\mathrm{P}<0.05)$, although MAD2 expression tended to be higher in group $\mathrm{BM}$ than in group $\mathrm{B}$ 
Table II. The weighted scores in groups B, BM and M.

\begin{tabular}{|c|c|c|c|c|c|c|}
\hline \multirow{3}{*}{$\begin{array}{l}\text { Weighted-score } \\
\\
0\end{array}$} & \multicolumn{6}{|c|}{ No. of cases } \\
\hline & \multicolumn{2}{|c|}{ Group B } & \multicolumn{2}{|c|}{ Group BM } & \multicolumn{2}{|c|}{ Group M } \\
\hline & 6 & 19 & 4 & 21 & 0 & 3 \\
\hline 1 & 7 & $(63.3 \%)$ & 8 & $(38.2 \%)$ & 0 & $(7.0 \%)$ \\
\hline 2 & 6 & & 9 & & 3 & \\
\hline 3 & 1 & 5 & 7 & 23 & 3 & 16 \\
\hline 4 & 2 & $(16.7 \%)$ & 9 & $(41.8 \%)$ & 3 & $(37.2 \%)$ \\
\hline 6 & 2 & & 7 & & 10 & \\
\hline 8 & 2 & 6 & 2 & 11 & 9 & 24 \\
\hline 9 & 3 & $(20.0 \%)$ & 4 & $(20.0 \%)$ & 11 & $(55.8 \%)$ \\
\hline 12 & 1 & & 5 & & 11 & \\
\hline Weighted score mean & 3.2 & & 4.3 & & 7.6 & \\
\hline
\end{tabular}
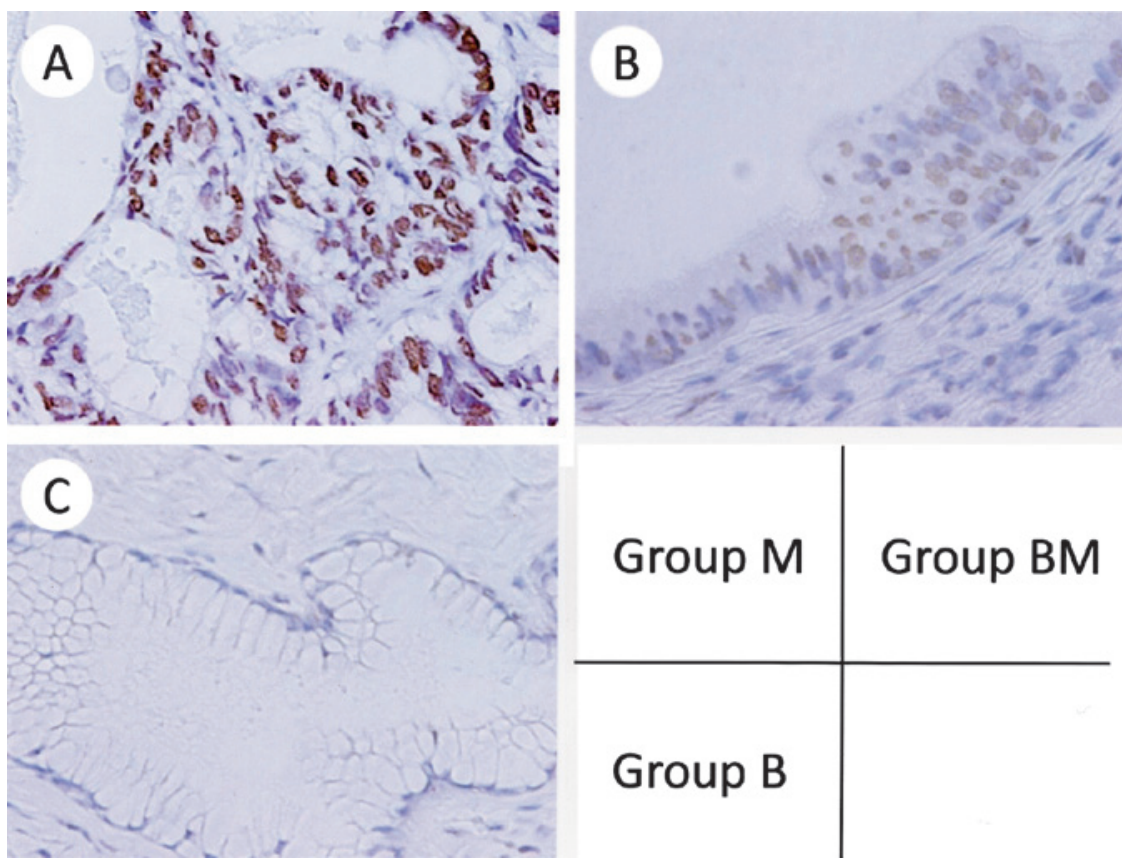

Figure 1. Immunohistochemical staining of MAD2 in mucinous ovarian tumors. (A) Group M, score 12; (B) Group BM, score 3; (C) Group B, score 0 (A-C, H\&E; magnification, $x 400)$. MAD2 was expressed in the nuclei of the tumor cells.

(Fig. 2). Thus, there was a moderate correlation between MAD2 expression and the degree of malignancy in mucinous ovarian tumors $(\mathrm{r}=0.51, \mathrm{P}<0.05)$.

Survival. A total of 43 cases in group $\mathrm{M}$ were classified as stage I $(n=25), 8$ as stage II and 10 as stage III/IV according to FIGO criteria. The overall survival for stage III/IV cases was significantly shorter than that for stages I and II (Fig. 3), with an estimated mean survival of 36 months versus 120 months and 117 months, respectively $(\mathrm{P}<0.05)$.

Correlation between clinical background and the expression of MAD2 in group M. A total of 43 cases within group $\mathbf{M}$ were sub-classified into low expression $(n=19)$ and high expression $(n=24)$ of MAD2. The weighted scores for the low expression group were 2-6, and those in the high expression group were 8-12. The mean age of the low expression group was 53.2 years (range 31-82) and that of the high expression group was 53.6 (range 23-71). Based on the FIGO criteria, the low expression cases were classified as stage I $(n=11)$, stage II $(n=6)$, stage III $(n=1)$ and stage IV $(n=1)$. The high expression cases were classified as stage I $(n=14)$, stage II $(n=2)$, stage III $(n=7)$ and stage IV $(n=1)$. Six patients within the low expression group relapsed, and 3 patients succumbed to the disease, whereas 4 patients within the high expression group relapsed and all of them succumbed to the disease 
Table III. Clinical background of cases in group $\mathrm{M}$ and a low and high expression of MAD2.

\begin{tabular}{lcc}
\hline & $\begin{array}{c}\text { Low expression } \\
\text { (score: } 2,3,4,6)\end{array}$ & $\begin{array}{c}\text { High expression } \\
\text { (score: } 8,9,12)\end{array}$ \\
\hline No. of cases & 19 & 24 \\
Age & & \\
Mean & 53.2 & 53.6 \\
Range & $31-82$ & $23-71$ \\
FIGO stage & & \\
I & 11 & 14 \\
II & 6 & 7 \\
III & 1 & 1 \\
IV & 1 & 115.8 \\
Overall survival & 100.6 & $1-140$ \\
Mean & $3-119$ & 4 \\
Range & 6 & 4 \\
Relapse & 3 & 7 \\
Mortality & & \\
\hline
\end{tabular}

FIGO, International Federation of Gynecology and Obstetrics.

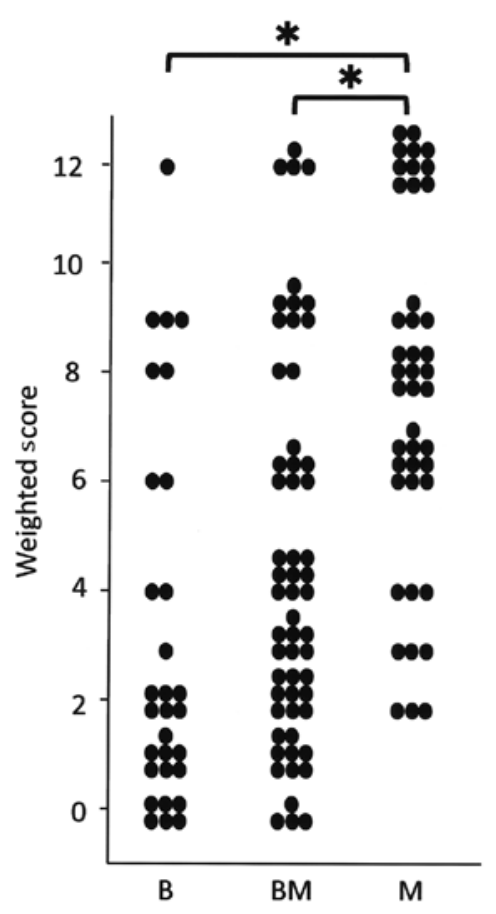

Figure 2. Weighted score for MAD2 in mucinous ovarian tumors is shown. MAD2 expression was significantly greater in group $M$ than in groups $B$ and BM. "P<0.05 (Mann-Whitney U test). MAD2 expression tended to be higher in $\mathrm{BM}$ than in group $\mathrm{B}(\mathrm{P}=0.064$, Mann-Whitney $\mathrm{U}$ test). Thus, there was a moderate correlation between MAD2 expression and the degree of malignancy in mucinous ovarian tumors $(r=0.51, \mathrm{P}<0.05$, Spearman's rank correlation coefficient).

(Table III). No difference was found between the low and high expression groups in terms of overall survival (Fig. 4), with an estimated mean survival time of 101 months versus 116 months $(\mathrm{P}=0.903)$.

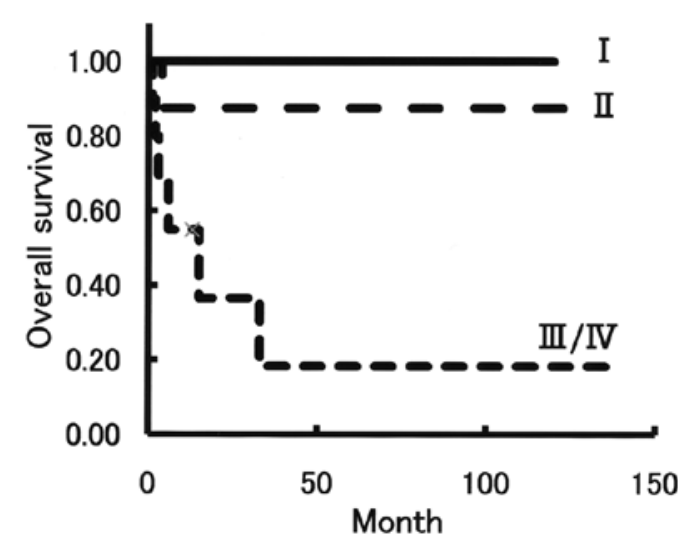

Figure 3. Overall survival according to FIGO criteria in group M. A total of 43 cases in group $M$ were sub-classified into stage $I(n=25)$, stage II $(n=8)$ and stage III/IV $(n=10)$ according to FIGO criteria. The overall survival for cases with stage III/IV was significantly shorter than that for cases with stage I and stage II ( $\mathrm{P}<0.05$, Kaplan-Meier and log-rank tests).

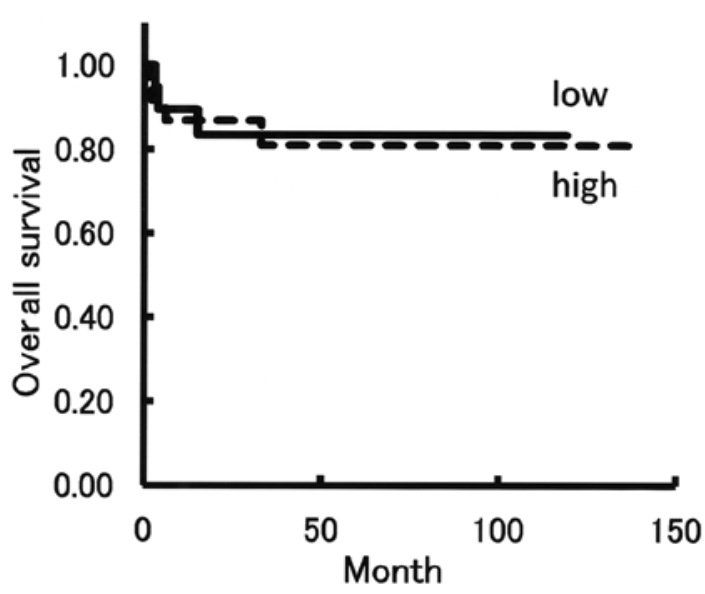

Figure 4. Overall survival according to the grade of MAD2 expression. The mean weighted scores in 43 cases in group $M$ was 7.6. These cases were sub-classified into low and high expression groups according to the weighted score. The low expression group had weighted scores from 2 to 6 , and the high expression group had weighted scores from 8 to 12 . There were 19 cases with a low expression and 24 with a high expression. No significant difference was observed between the low and high expression groups in terms of overall survival ( $\mathrm{P}=0.903$, Kaplan-Meier and log-rank tests).

\section{Discussion}

The aim of the present study was to investigate the correlation between MAD2 expression and carcinogenesis in mucinous ovarian tumors. The results show that MAD2 expression was significantly greater in group $M$ than in groups $B$ and BM. In addition, MAD2 expression tended to be higher in group BM than in group B (Fig. 2). Thus, there appears to be a moderate correlation between MAD2 expression and the degree of malignancy (Fig. 2). These findings suggest that the overexpression of MAD2 may be correlated to carcinogenesis in mucinous ovarian tumors. The benign epithelium histologically adjacent to the borderline tumor or carcinoma was also examined in the mucinous ovarian tumors, and the results have shown that MAD2 expression in the benign epithelium adjacent to the borderline tumor or carcinoma was higher than 
that in the original benign tumor. This suggests the possibility of a correlation between MAD2 expression and carcinogenesis in mucinous ovarian tumors.

A number of studies have emphasized the relationship between the overexpression of MAD2 and a variety of clinicopathological characteristics, including histologic grade (differentiation), metastasis and prognosis (14-20). Almost all of the authors of these studies have concluded that the overexpression of MAD2 is a risk factor for a high histologic grade, metastasis (e.g., to lymph nodes) and a poor prognosis. For example, 48 cases of human osteosarcoma were reviewed and the incidence of metastasis and survival time was assessed. MAD2 was expressed in all 48 cases. Cases were classified into three groups according to the mean percentage of MAD2-positive cells: (+) 25-49\%, (++) $50-74 \%$, and $(+++) 75-100 \%$. The median metastasis times for patients with a MAD2 score of $1+, 2+$ and 3+ were 62, 54 and 30 months, respectively. The median survival times for patients with a MAD2 score of $1+, 2+$ and 3+ were 75, 54 and 36 months, respectively. Overexpression of MAD2 was associated with early metastasis and a poor prognosis (19). In the present study, 48 cases in group $\mathrm{M}$ were sub-classified into low expression $(n=19)$ and high expression $(n=24)$ groups. The low expression group comprised cases with a weighted score from 2 to 6 , and the high expression group comprised cases with a weighted score from 8 to 12 . However, no difference was found between the low and high expression groups in terms of survival (Fig. 4). Moreover, the overexpression of MAD2 was not associated with any clinicopathological characteristics (Table III).

Findings of recent reports have shown that, once neoplastic transformation has occurred, the continued overexpression of MAD2 is no longer required for tumor maintenance (9). However, CIN induced by the overexpression of MAD2 leads to lung tumor relapse following oncogene withdrawal (21). Thus, the continued overexpression of MAD2 may be a risk factor for tumor progression and relapse. In the present study, 48 cases in group $M$ were sub-classified into relapse $(n=10)$ and non-relapse $(n=32)$ groups. We then examined the degree of MAD2 expression according to the weighted scores. No difference was found among the groups (data not shown). A total of 48 cases in group $\mathrm{M}$ were sub-classified according to FIGO criteria as follows: stage I $(n=25)$, stage II $(n=8)$, and stage III/IV $(\mathrm{n}=10)$. MAD2 expression was then examined according to the weighted scores. No statistically significant difference was observed among the groups (Mann-Whitney $\mathrm{U}$ test), although as observed for the stage III/IV, the expression of MAD2 was higher in stage I than in stage II (data not shown). The reason there was no significant difference is the small number of cases in group M, particularly stage III/IV cases. If more cases were included, the difference is likely to significant.

In conclusion, the results of the present study suggest that the overexpression of MAD2 is present in the majority of cases of mucinous ovarian carcinoma and may be correlated to the carcinogenesis of mucinous ovarian tumors. However, the overexpression of MAD2 may not in itself be sufficient for the carcinogenesis of mucinous ovarian tumors. Additionally, the degree of MAD2 expression was not associated with any clinicopathological characteristics.

\section{Acknowledgements}

We thank the gynecologists at Osaka City University Medical School Hospital for their support. This study was supported by the Osaka Medical Research Foundation for Incurable Diseases.

\section{References}

1. Hardwick KG: Checkpoint signaling: Mad2 conformers and signal propagation. Curr Biol 15: R122-R124, 2005.

2. Lopez-Girona A, Furnari B, Mondesert O and Russell P: Nuclear localization of Cdc25 is regulated by DNA damage and a 14-3-3 protein. Nature 397: 172-175, 1999.

3. Wang X, Jin DY, Ng RW, Feng H, Wong YC, Cheung AL and Tsao SW: Significance of MAD2 expression to mitotic checkpoint control in ovarian cancer cells. Cancer Res 62: 1662-1668, 2002.

4. Li R and Murray A: Feedback control of mitosis in budding yeast. Cell 66: 519-531, 1991.

5. Orr-Weaver TL and Weinberg RA: A checkpoint on the road to cancer. Nature 392: 223-224, 1998.

6. Yoon DS, Wersto RP, Zhou W, Chrest FJ, Garret ES, Kwon TK and Gabrielson E: Variable levels of chromosomal instability and mitotic spindle checkpoint defects in breast cancer. Am J Pathol 161: 391-397, 2002.

7. Lengauer C, Kinzler KW and Vogelstein B: Genetic instability in colorectal cancer. Nature 386: 623-627, 1997.

8. Lengauer C, Kinzler KW and Vogelstein B: Genetic instability in human cancer. Nature 396: 643-649, 1998.

9. Sotillo R, Hernando E, Diaz-Rodriguez E, Teruya-Feldstein J, Cordon-Cardo C, Lowe SW and Benezra R: Mad2 overexpression promotes aneuploidy and tumorigenesis in mice. Cancer Cell 11: 9-23, 2007.

10. To-Ho KW, Cheung HW, Ling MT, Wong YC and Wang X: $\mathrm{MAD} 2 \Delta \mathrm{C}$ induces aneuploidy and promotes anchorage-independent growth in human prostate epithelial cells. Oncogene 27: 347-357, 2008.

11. Alizadeh AA, Eisen MB, Davis RE, et al: Distinct types of diffuse large B-cell lymphoma identified by gene expression profiling. Nature 403: 503-511, 2000.

12. Chen X, Cheung ST, So S, et al: Gene expression patterns in human liver cancers. Mol Biol Cell 13: 1929-1939, 2002.

13. Garber ME, Troyanskaya OG, Schluens K, et al: Diversity of gene expression in adenocarcinoma of the lung. Proc Natl Acad Sci USA 98: 13784-13789, 2001.

14. Li GQ and Zhang HF: Mad2 and p53 expression profiles in colorectal cancer and its clinical significance. World J Gastroenterol 9: 1972-1975, 2003.

15. Li GQ and Zhang HF: Mad2 and p27 expression profiles in colorectal cancer and its clinical significance. World $\mathrm{J}$ Gastroenterol 10: 3218-3220, 2004.

16. Zhang SH, Xu AM, Chen XF, Li DH, Sun MP and Wang YJ: Clinicopathologic significance of mitotic arrest defective protein2 overexpression in hepatocellular carcinoma. Hum Pathol 39: 1827-1834, 2008.

17. Wang L, Yin F, Du Y, Du W, Chen B, Zhang Y, Wu K, Ding J, Liu J and Fan D: MAD2 as a key component of mitotic checkpoint: A probable prognostic factor for gastric cancer. Am J Clin Pathol 131: 793-801, 2009.

18. Tanaka K, Nishioka J, Kato K, Nakamura A, Mouri T, Miki C, Kusunoki M and Nobori T: Mitotic checkpoint protein hsMAD2 as a marker predicting liver metastasis of human gastric cancer. Jpn J Cancer Res 92: 952-958, 2001.

19. Yu L, Guo WC, Zhao SH, Tang J and Chen JL: Mitotic arrest defective protein 2 expression abnormality and its clinicopathologic significance in human osteosarcoma. APMS 118:222-9, 2010.

20. Wu CW, Chi CW and Huang TS: Elevated level of spindle checkprotein MAD2 correlates with cellular mitotic arrest, but not with aneuploidy and clinicopathological characteristics in gastric cancer. World J gastroenterol 10: 3240-3244, 2004.

21. Sotillo R, Schvartzman JM, Socci ND and Beneztra R: Mad2induced chromosome instability leads to lung tumor relapse after oncogene withdrawal. Nature 464: 436-40, 2010.

22. Scully RE, Young RH and Clement PB: Tumors of the ovary, maldeveloped gonads, fallopian tube and broad ligament. Atlas of tumor pathology, 3rd series, fascicle 23, Armed Forces Institute of pathology, Washington DC, 1998. 
23. Hart WR: Borderline epithelial tumors of the ovary. Mod Pathol 18: S33-50, 2005.

24. Lee KR and Scully RE: Mucinous tumors of the ovary: a clinicopathologic study 196 borderline tumors (of intestinal type) and carcinomas, including an evaluation of 11 cases with 'pseudomyxoma peritonei'. Am J Surg Pathol 28: 1311-1318, 2002.

25. Winter WE, Maxwell GL, Tian C, Carlson JW, Ozols RF Rose PG, Markman M, Armstrong DK, Muggia F and McGuire WP: Prognostic factors for stageIII epithelial ovarian cancer: a gynecologic oncology group study. J Clin Oncol 25: 3621-3627, 2007.

26. Puls LE, powell DE, DePriest PD, Gallion HH, Hunter JE, Kryscio RJ and van Nagell JR: Transition from benign to malignant epithelium in mucinous and serous ovarian cystadenocarcinoma. Gynecol Oncol 47: 53-57,1992.
27. Garrett AP, Lee KR, Colitti CR, Muto MG, Berkowitz RS and Mok SC: K-ras mutation may be an early event in mucinous ovarian tumorigenesis. Int J Gynecol Pathol 20: 244-251, 2001.

28. Wamunyokoli FW, Bonome T, Lee JY, Feltmate CM, Welch WR, Radonovich M, Pise-Masison C, Brady J, Hao K, Berkowitz RS, Mok S and Birrer MJ: Expression profiling of mucinous tumors of the ovary identifies genes of clinicopathologic importance. Clin Cancer Res 12: 690-700, 2006.

29. Sinicrope FA, Ruan SB, Cleary KR, Stephens LC, Lee JJ and Levin B: Bcl-2 and p53 oncoprotein expression during colorectal tumorigenesis. Cancer Res 55: 237-241, 1995. 